\title{
I 028 The hyperenhanced region assessed by ex vivo DE-MRI is consistently larger than infarct size determined by TTC staining in the acute phase of reperfused ischemic myocardial injury Andreas Otto-student*1, Henrik Engblom ${ }^{1}$, Einar Heiberg1 ${ }^{1}$, Martin Ugander ${ }^{1}$, Matthias Götberg2 ${ }^{2}$, Göran K Olivecrona ${ }^{2}$, David Erlinge ${ }^{2}$ and Håkan Arheden ${ }^{1}$
}

Address: ${ }^{1}$ Department of Clinical Physiology, Lund University, Lund, Sweden and ${ }^{2}$ Department of Cardiology, Lund University, Lund, Sweden * Corresponding author

from I It Annual SCMR Scientific Sessions Los Angeles, CA, USA. I-3 February 2008

Published: 22 October 2008

Journal of Cardiovascular Magnetic Resonance 2008, I0(SuppI I):AI53 doi:I0.II 86/I532-429X-I0-SI-AI53

This abstract is available from: http://jcmr-online.com/content/I0/SI/AI53

(c) 2008 Otto-student et al; licensee BioMed Central Ltd.

\section{Introduction}

The accuracy of DE-MRI measurement of myocardial infarct size in the acute phase has previously been investigated in different animal models. However, there is still an ongoing debate whether edematous reversible myocardial injury in the peri-infarction zone hyperenhances or not. If present, hyperenhancement of reversibly damaged myocardium will either lead to overestimation of the infarct size or indicate salvageable myocardium. The ability of DE-MRI to quantify myocardial infarct size has often been based on a slice to slice comparison between DEMRI and corresponding TTC.

\section{Purpose}

We wanted to evaluate the ability of ex vivo DE-MRI to depict and quantify acute myocardial necrosis on a global level using TTC as reference method in a closed chest pig model.

\section{Methods}

In 12 domestic pigs (40-50 kg), the left anterior descendant artery (LAD) was occluded immediately distal to the first diagonal branch for $40 \mathrm{~min}$, through inflation of an angioplasty balloon. Before explantation, the heart was reperfused for $4 \mathrm{~h} 22 \mathrm{~min} \pm 47 \mathrm{~min}$. Ex vivo DE-MRI was performed using a $1.5 \mathrm{~T}$ Philips Intera CV MR scanner. A gadolinium-based contrast agent (Gd-DTPA) was admin- istered intravenously $(0.2 \mathrm{mmol} / \mathrm{kg})$ both 60 and $15 \mathrm{~min}$ utes prior to removal of the heart. After removal, the heart was immediately rinsed in ice cold saline and the ventricles were filled with balloons containing deuterated water. Three-dimensional images with an isometric resolution of $0.5 \mathrm{~mm}$ covering the entire heart were acquired using T1weighted sequence (Fig 1). Subsequently, the hearts were sectioned into $4 \mathrm{~mm}$ consecutive slices in the short axis view, stained with TTC and scanned digitally (Fig 2). The short-axis ex vivo DE-MRI images were analyzed by manual delineation of the myocardium using freely available software (Segment 1.673, http://segment.heiberg.se). The infarct volume was calculated as the product of the slice thickness $(\mathrm{cm})$ and the area of hyperenhanced pixels $\left(\mathrm{cm}^{2}\right)$. Hyperenhancement was defined as $8 \mathrm{SD}$ above mean intensity of non-ischemic remote myocardium. The number of standard deviations was identified by using the k-means algorithm which agreed well with visual inspection. In the TTC stained slices, infarct size was determined by manual delineation of the endocardium, epicardium and TTC-negative area of the scanned images by an observer blinded to the ex vivo DE-MRI findings. Same software as described above was used for this purpose. Finally, the global amount of hyperenhancement assessed by DE-MRI and infarct size by TTC were calculated and expressed as percentage of left ventricular mass (LVM). 


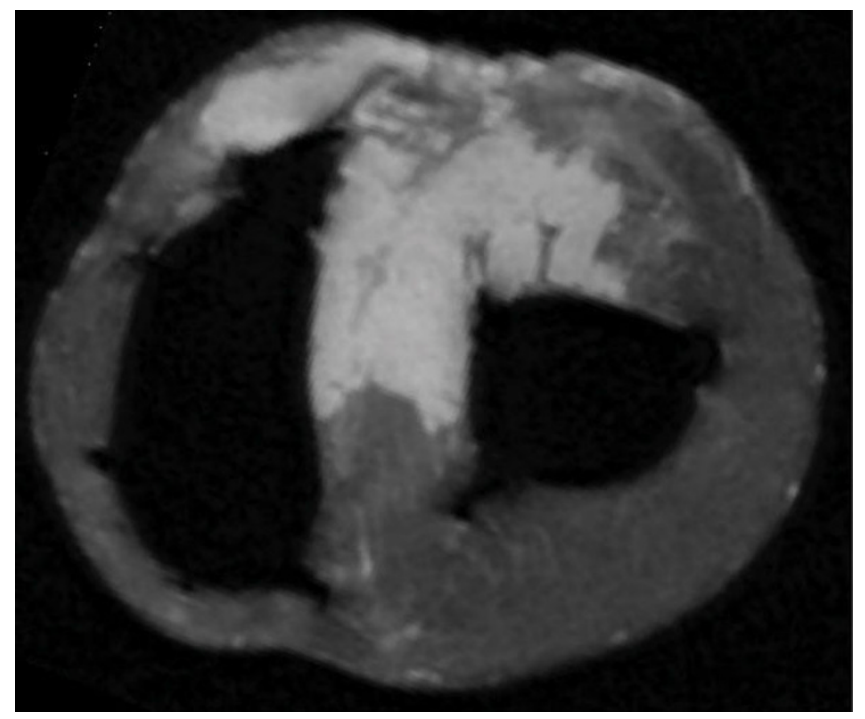

Figure I

A representative mid-ventricular ex vivo DE-MRI slice.

\section{Results}

There was a strong correlation between the global amount of hyperenhancement assessed by ex vivo DE-MRI and global infarct size determined by TTC histochemistry ( $\mathrm{y}=$ $1.27 \mathrm{x}, \mathrm{R}^{2}=0.93, \mathrm{p}<0.001$ ) (Fig 3). The hyperenhanced region measured $29 \pm 8 \%$ and the infarct size by TTC was $23 \pm 6 \%$ (mean difference, $6 \pm 2 \%$ ). Thus, the amount of hyperenhancement myocardium was $27 \%$ larger than the infarct size determined by TTC. Results are presented as mean percentage of $\mathrm{LVM} \pm \mathrm{SD}$.

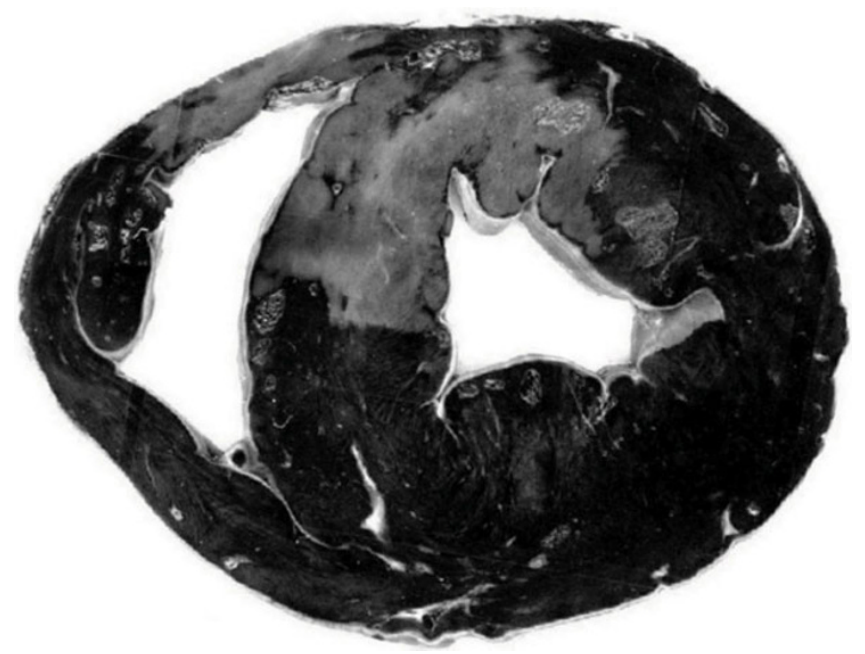

Figure 2

Corresponding TTC staining to the DE-MRI slice shown in Figure I.

\section{DE-MRI vs TTC}

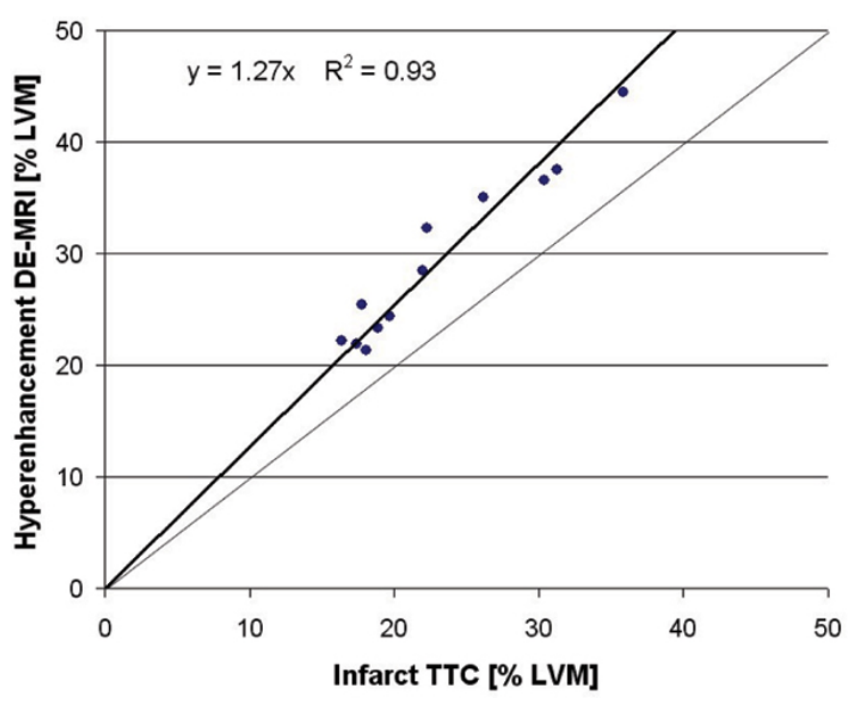

Figure 3

Statistical relationship between the amount of hyperenhancement by DE-MRI and infarct size by TTC. (LVM = left ventricular mass).

\section{Conclusion}

The global amount of hyperenhancement assessed by ex vivo DE-MRI is $27 \%$ larger than infarct size determined by TTC histochemistry in the acute phase. This may be attributed to hyperenhancement of both necrotic myocardium in the core of injury and injured but viable edematous myocardium in the peri-infarction zone. Thus, using hyperenhancement in DE-MRI as a definition of myocardial infarction will result in overestimation of myocardial infarct size in the acute phase of reperfused ischemic injury.

\section{Publish with Bio Med Central and every scientist can read your work free of charge}

"BioMed Central will be the most significant development for disseminating the results of biomedical research in our lifetime. " Sir Paul Nurse, Cancer Research UK

Your research papers will be:

- available free of charge to the entire biomedical community

- peer reviewed and published immediately upon acceptance

- cited in PubMed and archived on PubMed Central

- yours - you keep the copyright
BioMedcentral 